\section{Auricular Acupuncture as an Alternative Medicine}

\section{Gary Stanton* \\ Department of Neurology, Sleep Medicine and Pain Medicine Services,} Emerson Hospital, USA

\begin{abstract}
Auricular acupuncture has existed since antiquity for various symptomatic treatment purposes. Sixty-five years ago, Dr. Paul Nogier of France first theorized the treatment of the ear as a somatotopic microsystem, extensively developed its clinical utility, and named his treatment approach "auriculotherapy". His original method has since spread worldwide. Two major forms of auricular acupuncture consequently evolved, including European biomedical approach properly known as auriculotherapy; as opposed to an Asian Traditional Medicine approach. This report briefly summarizes the history of auricular acupuncture, its clinical rationale, indications, methods and advantages.
\end{abstract}

Keywords: Auricular acupuncture; Auriculotherapy

\section{Brief Report}

In 1952, Dr. Paul Nogier, a French physician, noted that some of his patients had similar small iatrogenic lesions on their ears. He questioned them, and found that each patient had seen a therapist, Madame Barrin, who had a reputation for being able to treat sciatica by cauterizing a specific spot on the auricle of the external ear. Having studied acupuncture previously, he knew that Traditional Chinese Acupuncture cartographies did not include points precisely on the ear, although the ear was believed to figure in the acupuncture meridian system, and that ear points were used occasionally as extra meridian treatment targets. In fact, auricular treatments have been used since antiquity in various regions, such as the Mediterranean basin, but were never developed systematically into a coherent therapeutic method. That was until Dr. Nogier began his research into the utility of treatment of the ear. He essentially rediscovered and advanced auricular acupuncture. The result was his identification of what he came to call "auriculotherapy" [1]. A German acupuncture journal publication of his results in Asia eventually came to the attention of Chinese acupuncturists, who confirmed the benefits of auricular acupuncture through their own independent research. In China and then worldwide Dr. Paul Nogier has been credited for the development of

${ }^{*}$ Corresponding author: Gary Stanton, Department of Neurology, Sleep Med icine and Pain Medicine Services, Emerson Hospital, 133 Old Road to Nine Acre Corner, JCB 600, Concord, MA 01742, USA, Tel: +1 9783718935; Fax: +1 9783718951; E-mail: gstanton@emersonhosp.org

Citation: Stanton G (2017) Auricular Acupuncture as an Alternative Medicine. J Altern Complement Integr Med 3: 023.

Received: January 23, 2017; Accepted: February 23, 2017; Published: March 09,2017 auriculotherapy as an original therapeutic method. In 1990, auriculotherapy was validated by the World Health Organization, and is now practiced throughout the world. In later years, Dr. Nogier went on to develop another approach to ear therapy, which he called "auriculomedicine". This is not included in the present discussion [2].

Two major approaches exist in auricular acupuncture. The original method of Dr. Nogier is called "auriculotherapy", as first developed by him in France. His work and auriculotherapy education are carried on in the organization he created, the Lyon Medical Studies Group (the French initials of which are "GLEM"). Instruction in auriculotherapy is also offered in some French medical schools, such as the University of Paris XI DIU degree program. The Eastern adaptation of auricular acupuncture, called by some "auriculopuncture", differs from the French approach. This is in part because the Asian ear acupuncture cartographies are somewhat different from European cartographies, and also because the Asian approach is largely based on the understanding of health and disease of Traditional Chinese Medicine, using concepts such as qi, yin, yang and five elements. In contrast, Dr. Nogier's approach to auriculotherapy was essentially biomedical, using concepts of neurophysiology. Therefore, diagnostic and treatment approaches differ to some extent between the occidental and oriental methods [2]. An example of a difference between Chinese and Franco-German cartographies using functional MRI was recently published [3]. The authors determined that the parts of the brain that react to Chinese auricular knee point stimulation are not exactly the same as the parts that react to the Franco-German knee point stimulation. Therefore, potentially significant neurophysiological and therefore clinical effects may be expected in choosing one cartography over another. Nevertheless, both approaches towards auricular acupuncture offer therapeutic benefits to patients.

Auricular acupuncture is increasingly researched worldwide, and results are published in medical journals. As a result, auricular acupuncture is now considered by many physicians to be an evidence based medicine. For example, a high quality randomized, blinded, controlled trial showing a statistically significant effect $(\mathrm{P}<0.0001)$ of auricular acupuncture in cancer pain has been published [4]. However, not all published literature is of high quality, and more research is needed [5]. In many United States acupuncture schools the Chinese approach is emphasized, as it is consistent with the principles of Traditional Chinese Medicine. However, instruction in the Nogier style auriculotherapy is also available in North America.

It is important to point out that one should be somewhat cautious in speaking about Western versus Eastern medicine in 2017. There are great biomedical centers in Asia and traditional medicine is practiced in Europe and the Americas. Both forms of medicine are global. In fact, much of the world's medical literature involving auricular acupuncture comes from Asia. I favor the approach of contrasting modern biomedicine with traditional medicine.

Dr. Nogier's great insight was that not only is the ear a valuable target for acupuncture, but that the organization of acupunctural target points on the ears follows a somatotopic pattern, unlike Traditional Chinese Meridian Theory. In Nogier's theory, specific cutaneous regions of the ear are correlated to specific regions of the body, internally 
and externally. For example, the area of the head and face are represented by potentially reactive acupuncture points in the earlobe. In the ear concha, located next to the external auditory canal are represented potential thoracic and abdominal visceral organ points. Above and posterior to this area are represented potential points of somatic structures such as the limbs and other elements of the nervous system. A way to understand this map of the ear is to imagine a drawing of a human being of fetal proportions inverted over the auricle of the ear. In fact, one of Nogier's publications was entitled "The Man in the Ear" [6]. A color reproduction of one example of a French auriculotherapy cartography, along with a description of the University of Paris XIII program developed by Dr. D Alimi may be consulted in a recent publication [7]. For comparison, one might consult an example of a published Chinese cartography [8]. It should be noted that there is no single universally accepted Nogier style or Chinese style auricular cartography. Even Nogier himself evolved his own cartographies in the course of his research. It is useful to study similarities and differences among auricular cartographies.

Most commonly, target points are treated with needles. However, as in traditional acupuncture, a variety of non needling methods are available to stimulate auricular target points. These include: electroauriculotherapy [9] (direct stimulation of skin acupoints, or stimulation of needles in acupoints); low level laser auriculotherapy [10]; thermal therapies (stimulation with non-moxibustion heat [11] or cold [12]); acupressure (originally called by Nogier "massage" [1]), weak and strong magnets; beads/pellets; other needling methods(press tacks, staple puncture, Spinex needles, aqua needle injection $[9,13])$; bleeding techniques [8]; and other light therapies (infrared LED; chromatic colors) [9]. Many of the latter methods have the advantage of being non invasive. Approaches without needles may be particularly useful in certain patient populations, such as pediatric patients, patients with transmissible infections, immunocompromised patients and special populations such as patients with cardiac valvulopathies or prostheses and increased risk for cardiac infections. However, it should be kept in mind that not all acupunctural point stimulation methods are clinically equivalent, a fact that is relevant when comparing clinical trials [14]. An example of a non invasive method of electroauriculotherapy for drug resistant epilepsy consists of trans auricular vagus nerve stimulation [15].

Nogier believed that the efficacy of auriculotherapy could be explained to a great extent by the external ear's innervation, which is relatively complex. According to his theory, nerves that innervate the skin of the ear convey information to and from specific regions of the brain and spinal cord. The primary nerves that innervate the skin of the ear include: the auriculotemporal branch of the trigeminal nerve, the auricular branch of the vagus nerve, and the greater auricular branch of the superficial cervical plexus. In associated regions of the central nervous system, these auricular pathways interface with other neural signal trafficking. By virtue of this interfacing of auricular and non auricular neural activity, a modulation of neural trafficking occurs along with other physiological effects potentially influencing homeostasis of the brain and body, and consequently a great variety of symptoms [2,9]. It is important to understand that according to Nogier's theory, the somatotopic representation of the body in the ear has an anatomic and physiologic correlation to the somatotopic organization of the central nervous system, the latter being a widely accepted tenet of neurophysiology. Auriculotherapy theory postulates that ear points become reactive as a result of underlying body pathology, which ultimately is detected and encoded in the brain's homeostasis.
Reactive ear points may be clinically detected by a variety of methods, such as probing for tenderness, or by testing for focal alteration of skin impedance using electro diagnosis. In treating ear points, one modulates the homeostatic activity in corresponding CNS somatotopic regions to reflexively effect therapeutic responses. The ability to treat the whole within the part has been likened to holography, which in itself represents an interesting hypothesis of neurophysiological organization $[2,16]$.

As with traditional acupuncture, the auricular acupuncture microsystem may thereby be applied to a wide variety of clinical situations. Common applications include treatment of anxiety, pain and insomnia, although there are many other potential indications for auriculotherapy. For example, a well known published application of auricular acupuncture is called the NADA protocol (the National Acupuncture Detoxification Association), which may be an effective adjunctive therapy for drug detoxification and behavioral health disorders [17]. The United States Military is interested in another well known auricular acupuncture protocol for pain known as "Battlefield Acupuncture" [18].

There are some advantages of auricular acupuncture over traditional acupuncture in the clinical setting, although both methods have merit, and in fact may be combined. Auricular acupuncture is convenient to apply, as it is unnecessary for a patient to disrobe. For physicians, auriculotherapy as developed by Nogier may be more readily understood than Traditional Chinese Medicine. This is because a medical student's understanding of biomedical principles is directly applicable to practicing auriculotherapy, whereas traditional medicine involves the study of a different concept of health and disease. Although the latter is interesting, logical coherent and useful, its mastery may be relatively challenging to someone trained in allopathic biomedicine. In fact, I believe that physicians who are interested in the world of acupuncture may do well to enter it through the French method of auriculotherapy. Another advantage of auriculotherapy is that Dr. Nogier developed a special $2 \mathrm{~mm}$ "semi permanent" needle with a mini barb at its end (called the "ASP" needle in France), which is placed in the skin of the external ear and retained for a longer period of time than in the traditional acupuncture standard needle treatment setting. Whereas the latter approach usually involves placing a needle in a point for 20-40 minutes, the ASP needle may safely remain in a point hours, days, even 1-2 weeks, eventually falling out spontaneously. This prolonged point stimulation may possibly confer an additional benefit beyond acute standard needling, and also reduces the need for a patient to return frequently for added treatments. A typical therapeutic trial of auriculotherapy may involve a 30 minute treatment session in which 3-5 small ASP needles are placed in one or both ears, once a month for three months. As with traditional acupuncture, the risk of infection or other complications of auricular acupuncture is quite low in competent hands. For patients whose acupuncture is not covered by health insurance, the diminished frequency of treatment visits helps keep down costs [2,9].

In conclusion, auriculotherapy treatments are relatively conservative, safe, often helpful and cost effective. Auriculotherapy is now considered an evidence based medicine for which high quality scientific evidence has been published. More research is needed, however, and readers are encouraged to engage in such research. 


\section{References}

1. Nogier PMF (1998) Handbook to Auriculotherapy (2ndedn). Satas, Brussels, Belgium.

2. Rouxeville Y, Meas Y, Bossy J (2007) Auriculotherapy Auricular Acupuncture [in French]. Springer-Verlag France, Paris.

3. Alimi D, Geissman A, Gardeur D, Bahr F (2014) Study in fMRI of the stimulation of the auricular areas of the knee as the French-German and Chinese localizations. The Journal of Radiology, Photon 125: 133-141.

4. Alimi D, Rubino C, Pichard-Léandri C, Fermand-Brulé S, Dubreuil-Lemaire $\mathrm{ML}$, et al. (2003) Analgesic effect of auricular acupuncture for cancer pain: a randomized, blinded, controlled trial. J Clin Oncol 21: 4120-4126.

5. Lee H, Park HJ, Park J, Kim MJ, Hong M, et al. (2007) Acupuncture application for neurological disorders. Neurol Res 29: 49-54.

6. Nogier PMF (1979) L'Homme dans l'Oreille [in French]. Maisonneuve, Moulins les Metz, France.

7. Stanton G, Rangon CM (2014) The Scientific Auriculotherapy Diploma Program of the Universities of Paris XI and XIII. Med Acupuncture 26: 118-124.

8. Abbate S (2004) Chinese Auricular Acupuncture. CRC Press, Boca Raton, USA.

9. Rouxeville $Y$ (2016) Les clés de l'auriculothérapie, clinique et pratique [in French]. Satas, Brussels, Belgium.
10. Round R, Litshcer G, Bahr F (2013) Auricular Acupuncture with Laser. Evidence-based Compl and Alt Med. 2013: 984763.

11. http://www.sedatelec.com/english/cadre.htm

12. Alimi D (2017) Unpublished data. Personal communication.

13. Oleson T (1998) Auriculotherapy Manual: Chinese and Western Systems of Ear Acupuncture, 2nd edition. Los Angeles: Health Care Alternatives, Inc., Chicago, USA

14. Langevin HM, Schnyer R, MacPherson H, Davis R, Harris RE, et al. (2015) Manual and electrical needle stimulation in acupuncture research: pitfalls and challenges of heterogeneity. J Altern Complement Med 21: 113-128.

15. Rong P, Liu A, Zhang J, Wang Y, Yang A, et al. (2014) An alternative therapy for drug-resistant epilepsy: transcutaneous auricular vagus nerve stimulation. Chin Med J (Engl) 127: 300-304.

16. Frank BL, Soliman NE (2005) Auricular Therapy: A Comprehensive Text: Auricular Phases, Frequencies, and Blockages. Author House, Bloomington, Indiana, USA.

17. Stuyt EB, Voyles CA (2016) The National Acupuncture Detoxification Association protocol, auricular acupuncture to support patients with substance abuse and behavioral health disorders: current perspective. Subst Abuse Rehabil 7: 169-180.

18. Niemtzow RC (2007) Battlefield Acupuncture. Med Acupuncture 19: 225-228. 